NHCU-HEP-94-20

hep-ph/9506295

\title{
Axion-photon Couplings in Invisible Axion Models
}

\author{
S. L. Cheng ${ }^{a}$, C. Q. Geng ${ }^{b}$ and W.-T. Ni ${ }^{b}$ \\ ${ }^{a}$ Department of Physics, National Chung Hsing University \\ Taichung, Taiwan, Republic of China \\ and \\ ${ }^{b}$ Department of Physics, National Tsing Hua University \\ Hsinchu, Taiwan, Republic of China
}

\begin{abstract}
We reexamine the axion-photon couplings in various invisible axion models motivated by the recent proposal of using optical interferometry at the ASST facility in the SSCL to search for axion. We illustrate that the assignment of $U(1)_{P Q}$ charges for the fermion fields plays an important role in determining the couplings. Several simple non-minimal invisible axion models with suppressed and enhanced axionphoton couplings are constructed, respectively. We also discuss the implications of possible new experiments to detect solar axions by conversion to $X$-rays in a static
\end{abstract}


magnetic apparatus tracking the sun. 


\section{Introduction}

A interesting problem in the standard model is the strong $\mathrm{CP}$ problem [1] of why the parameter $\theta$ which involves a $P$ and $T$-odd term in the QCD Lagrangian, is so small: $\theta<10^{-9}$. A natural and elegant solution to this problem is the Peccei-Quinn (PQ) mechanism [2] which yields $\theta=0$ dynamically. The spontaneous breakdown of chiral global $U(1)_{P Q}$ symmetry gives rise to a pseudo-Goldstone boson called axion [3]. To elude current experimental detections it has to be very light and weakly coupled and therefore is dubbed the "invisible" axion. Astrophysical and cosmological considerations require its mass $m_{a}$ to lie between $10^{-3}$ and $10^{-6} \mathrm{eV}$ [1].

Recent observation of the quadrupole anisotropy in the cosmic microwave background radiation [4, 5], favors cold dark matter cosmologies [6]. Axions and/or other pseudo-Goldstone bosons together with light mass neutrinos are potentially good candidates for cold dark matter. Interests in axion search have been enhanced recently.

There are existing many interesting ongoing and underway experiments to search for the axion [1, 7], especially the recent proposal [8] of using optical interferometry at the Accelerator System String Test (ASST) facility in the SSCL to study the velocity of light in a strong magnetic field. Since the axion couples to photons through the anomaly, the magnitude of the light retardation in a magnetic field depends on the axion mass and its coupling strength, which are model-dependent. Measuring a deviation from the QED prediction would test and distinguish various invisible axion models. The sensitivity of the experiments is related to the accuracy of the measurement of the vacuum birefringence effect. For the accuracy of $0.3 \%$ expected from the ultra-high sensitive interferometer the search for axions can reach $10^{11} \mathrm{GeV}$ 
for the inverse of the axion-photon coupling, which is several orders of magnitude higher than that reached by previous experiments [8]. More specifically, the proposed experiment aims at using SSCL ASST $170 m$ magnet string facility with a dipole field of 6.6 T. Using a Fabry-Perot cavity of laser $(\lambda=1.06 \mu \mathrm{m})$ of the output power $P=50 \mathrm{~W}$, the sensitivity limited by the shot noise is $2.64 \times 10^{-20} \mathrm{~m} / \sqrt{\mathrm{Hz}}$ for the optical pathlength variation $(\delta l)$ due to different polarization and $1.70 \times 10^{-22} / \sqrt{H z}$ for the variation of refraction index $(\delta n)$ due to different polarization with active length $155 \mathrm{~m}$. For an integration time of about 30 hours, $\Delta f=10 \mu \mathrm{Hz}$, it is possible to measure $\delta n$ to $5.38 \times 10^{-25}$. In a field of $6.6 \mathrm{~T}$ magnetic field, $\delta n$ is $1.75 \times 10^{-22}$. Hence, for an integration of 30 hours, the QED birefringence effect would be detected to $0.3 \%$. With this sensitivity, the mass scale $M$ that can be probed for axions approaches $10^{11} \mathrm{GeV}$. Longer integration will give better sensitivity.

The most recent search for solar axion [9] is to exploit their conversion to $X$-rays in a static magnetic field for detection. They used a magnet of length $1.8 \mathrm{~m}$ and field strength $B=2.2 \mathrm{~T}$. The diameter of the axion converter is about $0.15 \mathrm{~m}$. Data were taken for about two hours centered around sunset of two days. The effective time window is about 15 mins per day. They set a limit of $M>2.79 \times 10^{8} \mathrm{GeV}$ (99.7\% C.L.) for $m_{a}<0.03 \mathrm{eV}$. With a longer magnet $(5 \mathrm{~m})$ and longer effective diameter $(3 \mathrm{~m})$ at $7 \mathrm{~T}$ tracking the sun, the sensitivity for detecting axions can be improved by two orders of magnitude or more in $M$. Building such a magnet is feasible. As a matter of fact, among the two superconducting electromagnetic thrusters for the superconducting electromagnetic propulsion ship YAMATO1 (experimental ship), [t each has six coils arranged in a circle. The coils have an inside diameter of

\footnotetext{
${ }^{a}$ We thank M. Wake for providing the information of the YAMATO1 superconducting magnets to us.
} 
$0.36 \mathrm{~m}$ and effective length of $3 \mathrm{~m}$ with a magnetic field $3-4 \mathrm{~T}$. The total volume for the magnetic field of each thruster is $1.8 \mathrm{~m}^{3}$. Therefore, the mass scale $M$ that can be probed for solar axion conversion approaches $10^{11} \mathrm{GeV}$. Larger volume and higher field or better detector sensitivity and longer integration will give better sensitivity. This method is comparable to the optical interferometry method in sensitivity to $M$. Recently, an experimental effort has been going on in Novosibirsk by using a gimballed magnet to track the Sun with a sensitivity goal approaching $10^{10} \mathrm{GeV}[10$.

In this paper, we will systematically reexamine the axion-photon couplings in axion models. In particular, we will explore the possibility of having small or large couplings in simple extensions of the original invisible axion models.

\section{The models}

There are three minimal types of invisible axion models: (1) Dine-FischlerSrednicki-Zhitiniskii (DFSZ) type [11], which has two doublets $\phi_{i}(i=1,2)$ and one singlet $\chi$ Higgs fields; (2) Kim-Shifman-Vainshtein-Zakharov (KSVZ) type [12, which contains one $\phi$ and one $\chi$ Higgs fields plus a superheavy exotic $S U(2) \times U(1)$ singlet quark $Q$; and (3) the variant invisible axion (VIA) models 13. The latter modifies the variant weak scale axion models [14] by adding a single Higgs field as the DFSZ models. There are many variations of these minimal axion models depending on the choices of the PQ charges.

Without loss of generality, we assign the following $U(1)_{P Q}$ transformations for the Higgs and the fermion fields:

$$
\phi_{1} \rightarrow \exp \left(-i h_{1}\right) \phi_{1}, \phi_{2} \rightarrow \exp \left(i h_{2}\right) \phi_{2}
$$




$$
\begin{aligned}
& \chi \rightarrow \exp \left(-i\left(h_{1}+h_{2}\right) / 2\right) \chi, \\
& d_{R}^{j} \rightarrow \exp \left(i X_{d}^{j}\right) d_{R}^{j}, u_{R}^{j} \rightarrow \exp \left(i X_{u}^{j}\right) u_{R}^{j}, e_{R}^{j} \rightarrow \exp \left(i X_{e}^{j}\right) e_{R}^{j},
\end{aligned}
$$

in the DFSZ and VIA models with $j=1, \cdots, N_{g}$ being the generation index, and

$$
\begin{aligned}
& \chi \rightarrow \exp \left(i Q_{P Q}\right) \chi \\
& Q_{L} \rightarrow \exp \left(i Q_{P Q} / 2\right) Q_{L}, Q_{R} \rightarrow \exp \left(-i Q_{P Q} / 2\right) Q_{R},
\end{aligned}
$$

in the KSVZ models. The PQ transformations in the DFSZ models are generation blind and they distinguish only the up- and down-type quarks and charged leptons. Thus, in these models, we have

$$
\begin{aligned}
& X_{u} \equiv X_{u}^{i}, X_{d} \equiv X_{d}^{i}, X_{e} \equiv X_{e}^{i}, \\
& X_{u} \neq-X_{d}
\end{aligned}
$$

with $i=1, \cdots, N_{g}$. The down-type (up-type) quarks can get masses from the Yukawa interactions by choosing $h_{1(2)}=X_{d(u)}$. However, there is a freedom for the lepton Yukawa couplings, depending on the PQ charge of $X_{e}$. This freedom leads to two minimal DFSZ models:

$$
\begin{array}{cl}
\text { DFSZ I }: & X_{e}=X_{d}, \\
\text { DFSZ II }: & X_{e}=-X_{u} .
\end{array}
$$

The first model is the original DFSZ model. We now consider the cases that the charged lepton masses arise from a third Higgs doublet, $\phi_{3}$, to take into account the lightness of their masses comparing with the quark ones. We assign the PQ charge of $\phi_{3}$ as

$$
h_{3}=-X_{e} \neq\left|X_{u}\right|,\left|X_{d}\right| .
$$


We refer the assignment in Eq. (5) as DFSZ III. For the VIA models, $U(1)_{P Q}$ is no longer family blind. As an example, we take $N_{g}=3$ and the PQ charges in Eq. (1) as

$$
\begin{aligned}
& \left(X_{u}^{1}, X_{u}^{2}, X_{u}^{3}\right)=\left(-X_{d},-X_{d}, X_{u}\right), \\
& \left(X_{d}^{1}, X_{d}^{2}, X_{d}^{3}\right)=\left(X_{d}, X_{d}, X_{d}\right), \\
& \left(X_{e}^{1}, X_{e}^{2}, X_{e}^{3}\right)=\left(X_{d}, X_{d}, X_{d}\right) .
\end{aligned}
$$

In this VIA model, the fact that the top quark is much heavier than other flavors could be understood if $v_{1} \ll v_{2}$, where $v_{1(2)}$ is VEV of $\phi_{1(2)}$. Finally we recall the composite axion model suggested by Kim [15]. In this model the transformations of the exotic Dirac fermions, under the group $S U(N)_{M C} \times S U(3)_{C} \times U(1)_{Y} \times U(1)_{P Q}$ are chosen to be

$$
\begin{gathered}
(N, 3, a ; 1)+(N, 1, b ;-3) \\
(\bar{N}, \overline{3},-a ; 1)+(\bar{N}, 1,-b ;-3),
\end{gathered}
$$

where $N$ is the dimension of the metacolor representation. Clearly, $U(1)_{P Q}$ is indeed a PQ symmetry since it has a color anomaly.

\section{The axion-photon interactions}

We write the effective axion-photon Lagrangian as

$$
\mathcal{L}_{a \gamma \gamma}=\frac{1}{4} g_{a \gamma \gamma} a F_{\mu \nu} \tilde{F}^{\mu \nu}
$$

where $F_{\mu \nu}$ is the electromagnetic field tensor and $\tilde{F}^{\mu \nu}$ its dual, $a$ the axion field, and $g_{a \gamma \gamma}$ the coupling constant. For a static magnetic field and real photon, the 
interaction becomes

$$
\mathcal{L}_{a \gamma \gamma}=\frac{1}{M} a \vec{E} \cdot \vec{B}_{e x t}
$$

where $\vec{E}$ and $\vec{B}_{\text {ext }}$ are the electric and magnetic fields and $M$ is the energy scale defined as the inverse coupling constant,

$$
M \equiv \frac{1}{g_{a \gamma \gamma}}
$$

which has dimensions of energy. Clearly, the axion only interacts with photon wave component that is parallel to an external magnetic field. One finds [16]

$$
g_{a \gamma \gamma}=\frac{\alpha m_{a}}{2 \pi f_{\pi} m_{\pi}} \frac{1+Z}{\sqrt{Z}}\left[\frac{A_{P Q}^{e m}}{A_{P Q}^{C}}-\frac{2}{3} \frac{(4+Z)}{(1+Z)}\right]
$$

where $Z \equiv m_{u} / m_{d}$ and $A_{P Q}^{e m}$ and $A_{P Q}^{C}$ are the anomaly factors given by

$$
\begin{aligned}
A_{P Q}^{e m} & =\operatorname{Tr} Q_{e m}^{2} Q_{P Q} \\
\delta_{a b} A_{P Q}^{C} & =\operatorname{Tr} \lambda_{a} \lambda_{b} Q_{P Q}
\end{aligned}
$$

with $\lambda_{a}$ being the color generators and $\operatorname{Tr} \lambda_{a} \lambda_{b}=\frac{1}{2} \delta_{a b}$. Using $Z \simeq 0.56, f_{\pi}=$ $93 \mathrm{MeV}$, and $m_{\pi}=134 \mathrm{MeV}$, we get

$$
g_{a \gamma \gamma}=1.36 \times 10^{-11} \frac{\alpha}{m_{e}}\left(\frac{m_{a}}{1 e V}\right)\left[\frac{A_{P Q}^{e m}}{A_{P Q}^{C}}-1.95\right]
$$

We note that the ratio $R \equiv A_{P Q}^{e m} / A_{P Q}^{C}$ is model-dependent. The coupling $g_{a \gamma \gamma}$ becomes minimal when $R$ is close to 1.95 and, on the other hand, it can be large for the ratio $R$ being negative or large.

We now examine the axion-photon coupling in the various invisible axion models given in the previous section. We are particularly interested in finding the cases that could result in small or large $g_{a \gamma \gamma}$. From Eq. (1), we find for the DFSZ and VIA 
models

$$
\begin{aligned}
& A_{P Q}^{e m}=\sum_{j}\left(\frac{4}{3} X_{u}^{j}+\frac{1}{3} X_{d}^{j}+X_{e}^{j}\right) \\
& A_{P Q}^{C}=\frac{1}{2} \sum_{j}\left(X_{u}^{j}+X_{d}^{j}\right) .
\end{aligned}
$$

This leads to

$$
R_{D F S Z}=\frac{2}{3}\left(4 X_{u}+X_{d}+3 X_{e}\right) /\left(X_{u}+X_{d}\right)
$$

for the DFSZ models and

$$
R_{V I A}=8 / 3
$$

for the VIA model in Eq. (6). For the DFSZ I and II and VIA models, the values of $R$ are fixed and the couplings are given by

$$
g_{a \gamma \gamma}=(1.4,-2.6,1.4) \times 10^{-10}\left(\frac{m_{a}}{1 e V}\right) G e V^{-1},
$$

respectively. However, $R$ in the DFSZ III varies as we change $X_{e}$ assignment. It is easily seen that a model with $R=2$ can be constructed if we chose

$$
X_{e}=1 / 3, \quad X_{u}=X_{d}=1
$$

in which we get

$$
g_{a \gamma \gamma}=9.9 \times 10^{-12}\left(\frac{m_{a}}{1 e V}\right) G e V^{-1},
$$

from Eqs. (13) and (15). We can also have models with large $g_{a \gamma \gamma}$. For example, if we set

$$
X_{e}=-1, \quad X_{u}=-1 / 3, \quad X_{d}=2 / 3,
$$


corresponding to $R=-22 / 3$, we get

$$
g_{a \gamma \gamma}=-1.9 \times 10^{-9}\left(\frac{m_{a}}{1 e V}\right) G e V^{-1}
$$

The absolute value of $g_{a \gamma \gamma}$ in Eq. (21) is about a factor of 200 larger than that in Eq. (19). The inverse axion-photon coupling strength $M \equiv g_{a \gamma \gamma}^{-1}$ in various cases are shown in Table 1.

Similarly, we have 16]

$$
R=6 Q_{e m}^{2} \text { and } 6\left(a^{2}-b^{2}\right)
$$

for the KSVZ and composite axion models, respectively. Here $Q_{e m}$ is the electric charge of the heavy quark in the KSVZ models. As emphasized by Kaplan in Ref. [16], due to the arbitrariness of the charges in Eq. (22) it is possible to create $R=2$ in the composite axion model naturally. As completeness we list it as well as other cases in Table 1.

As we can see from Table 1, it is impossible to detect the axion in the models with suppressed photon-axion couplings even with the new proposal of using optical interferometry at SSCL ASST facility as well as the improved experiment of $X$-ray conversion of solar axions. However, it could be accessible for the models which have enhanced photon couplings when further improvement on sensitivity is made.

\section{Conclusions}

In the light of the recent proposal of using interferometry at SSCL ASST facility to search for axions and pseudoscalar particles [8], we have systematically studied the axion-photon couplings in various invisible axion models. We have demonstrated that $g_{a \gamma \gamma}$ depends strongly on the assignment of $U(1)_{P Q}$ charges, and found that 
simple extensions of the minimal DFSZ models could lead to suppressed as well as enhanced axion-photon couplings.

Although the new proposal [8] aims at five orders of magnitude improvement on the sensitivity of the energy scale $M$, it is still not enough to detect the axions even in the models with enhanced photon-axion couplings. It may be marginal for the searches when further improvements on the sensitivity are made. The situation is similar for the method of $X$-ray conversion of solar axions. If the experiment finds deviations from the Quantum Electrodynamics at the initial stage, the effects must be new and not from the invisible axion models considered. Finally, we remark that with five orders of magnitude improvement, the regions of interest are rather wide. Critical examinations in other pseudo-scalar/scalar models as well as those with potential weak two-photon couplings would be mostly interesting.

\section{Acknowledgments}

This work was supported in part by the National Science Council of Republic of China under Grants NSC-83-0208-M-007-118 (C.Q.G) and NSC-83-0208-M-007-126 (W.-T.N). 


\section{References}

[1] For reviews, see J.E. Kim, Phys. Rep. 150 (1987) 1; H.Y. Cheng, Phys. Rep. 158 (1988) 1; R.D. Peccei, in CP Violation, ed. by C. Jarlskog, (World Scientific, Singapore, 1989), p. 503; M.S. Turner, Phys. Rep. 197 (1990) 67; G.G. Rafelt, Phys. Rep. 198 (1990) 1; and references therein.

[2] R.D. Peccei and H.R. Quinn, Phys. Rev. Lett. 38 (1977) 1440; Phys. Rev. D 16 (1977) 1791.

[3] S. Weinberg, Phys. Rev. Lett. 40 (1978) 223; F. Wilczek, Phys. Rev. Lett. 40 (1978) 279 .

[4] G. F. Smoot et al.,Astrophys. J. 396 (1992) L1.

[5] S. Hancock et al., Nature (London) 367 (1994) 333.

[6] R. L. Davis, Phys. Rev. Lett. 69 (1992) 1856.

[7] G. Cantatore et al., Nucl. Phys. B (Proc. Suppl.) 35 (1994) 180.

[8] Cf. "Light Retardation in a High Magnetic Field and Search for Light Scalar/Pseudo-scalar Particles Using Ultra-sensitive Interferometry", Joint Proposal Submitted to the National Science Council of the Republic of China and Department of Energy of the United States of America (April, 1994); see also W.-T. Ni et al., "Test of Quantum Electrodynamics and Search for Light Scalar/Pseudoscalar Particles Using Ultra-high Sensitive Interferometers", NHCU-HEP-94-12, in Proceedings of the Meeting on General Relativity, July 23-30, 1994, Stanford, CA (World Scientific, Singapore, 1995) (in press). 
[9] D. M. Lazarus et al., Phys. Rev. Lett. 69 (1992) 2333.

[10] P.V. Vorobyov and I.V. Kolokolov, "Detectors for the Cosmic Axionic Wind", ASTRO-PH-9501042 (1995).

[11] M. Dine, W. Fischler and M. Srednicki, Phys. Lett. B104 (1981) 199; A.P. Zhitnitskii, Sov. J. Nucl. Phys. 31 (1980) 260.

[12] J.E. Kim, Phys. Rev. Lett. 43 (1979) 103; M.A. Shifman, A.I. Vainshtein, and V.I. Zakharov, Nucl. Phys. B166 (1980) 493.

[13] C. Q. Geng and J. N. Ng, Phys. Rev. D39 (1989) 1449.

[14] R.D. Peccei, T.T. Wu and T. Yanagida, Phys. Lett. B172 (1986) 435; L.M. Krauss and F. Wilczek, Phys. Lett. B173 (1986) 189.

[15] J.E. Kim, Phys. Rev. D31 (1985) 1733.

[16] W.A. Bardeen and S.-H.H. Tye, Phys. Lett. B74 (1978) 229; D.D. Kaplan, Nucl. Phys. B260 (1985) 215; M. Srednicki, Nucl. Phys. B260 (1985) 689. 
Table 1: The inverse axion-photon coupling strength $M$ in invisible axion models

\begin{tabular}{|c|c|c|}
\hline Models & $\left(\frac{m_{a} \mathrm{eV}}{10^{-5}}\right)\left(\frac{M \mathrm{GeV}}{10^{14}}\right)$ & Remark \\
\hline DFSZ I & 7.1 & $X_{e}=X_{d}$ \\
\hline DFSZ II & 3.9 & $X_{e}=-X_{u}$ \\
\hline \multirow[t]{3}{*}{ DFSZ III } & 17 & $X_{e}=0, X_{u}=X_{d}$ \\
\hline & 101 & $X_{e}=1 / 3, X_{u}=X_{d}=1$ \\
\hline & 0.54 & $X_{e}=-1, X_{u}=-1 / 3, X_{d}=2 / 3$ \\
\hline VIA & 7.1 & \\
\hline \multirow[t]{4}{*}{ KSVZ } & 7.1 & $Q_{e m}=2 / 3$ \\
\hline & 3.9 & $Q_{e m}=-1 / 3$ \\
\hline & 1.2 & $Q_{e m}=1$ \\
\hline & 2.6 & $Q_{e m}=0$ \\
\hline \multirow[t]{2}{*}{ Composite } & 0.63 & $a=0, b=1$ \\
\hline & 101 & $a=2 / 3, b= \pm 1 / 3$ \\
\hline
\end{tabular}

* We take the absolute value of $M$. 